\title{
Finite-Time $l_{1}$-Gain Control for Positive Switched Systems with Time-Varying Delay via Delta Operator Approach
}

\author{
Shuo Li, ${ }^{1}$ Zhengrong Xiang, ${ }^{1}$ and Hamid Reza Karimi ${ }^{2}$ \\ ${ }^{1}$ School of Automation, Nanjing University of Science and Technology, Nanjing 210094, China \\ ${ }^{2}$ Department of Engineering, Faculty of Engineering and Science, University of Agder, 4898 Grimstad, Norway \\ Correspondence should be addressed to Zhengrong Xiang; xiangzr@mail.njust.edu.cn
}

Received 21 November 2013; Accepted 14 December 2013; Published 23 January 2014

Academic Editor: Hui Zhang

Copyright (c) 2014 Shuo Li et al. This is an open access article distributed under the Creative Commons Attribution License, which permits unrestricted use, distribution, and reproduction in any medium, provided the original work is properly cited.

\begin{abstract}
This paper is concerned with the problem of finite-time $l_{1}$-gain control for positive switched systems with time-varying delay via delta operator approach. Firstly, sufficient conditions which can guarantee the $l_{1}$-gain finite-time boundedness of the underlying system are given by using the average dwell time approach and constructing an appropriate copositive type Lyapunov-Krasovskii functional in delta domain. Moreover, the obtained conditions can unify some previously suggested relevant results seen in literature of both continuous and discrete systems into the delta operator framework. Then, based on the results obtained, a state feedback controller is designed to ensure that the resulting closed-loop system is finite-time bounded with an $l_{1}$-gain performance. Finally, a numerical example is presented to demonstrate the effectiveness and feasibility of the proposed method.
\end{abstract}

\section{Introduction}

Positive systems are dynamical systems where the states and outputs are constrained to be nonnegative whenever the initial conditions and inputs are nonnegative $[1,2]$. Such systems are applied in various areas, for instance, biomedicine $[3,4]$, ecology [5], TCP-like Internet congestion control [6, 7], industrial engineering [8], and so on. Recently, positive switched systems, which consist of a family of positive subsystems and a switching signal coordinating the operation of various subsystems to govern the switching among them, have been highlighted and investigated by many researchers due to the broad applications in communication systems $[7,9]$, formation flying [10], the viral mutation dynamics under drug treatment [2], and systems theories [11-13]. It has been shown that a linear copositive Lyapunov functional is powerful for the analysis and synthesis of positive systems [14-16].

The delta operator, as a novel method of solving the problem of system instability under fast sampling, has drawn considerable attention in the past three decades. As we know, most discrete-time signals and systems are the results of sampling continuous-time signals and systems. When the sampling period tends to zero, namely, data are taken at high sampling rates, however, all resulting signals and systems tend to be ill-conditioned and thus difficult to deal with using the conventional algorithms. Until Goodwin et al. proposed a delta-operator-based algorithm in [17] to take place of the conventional algorithms, the above problem is avoided. It was shown that delta-operator-based algorithms are numerically better behaved under finite precision implementations for fast sampling [17]. The delta operator model can be regarded as a useful approach to deal with discrete-time systems under high sampling rates through the analysis methods of continuous-time systems [18-20]. Based on significant early investigations such as [21-23], the numerical properties and practical applications of the delta operator model have been extensively investigated [24-26]. The delta operator is defined by

$$
\delta x(t)= \begin{cases}\frac{d x(t)}{d t}, & T=0 \\ \frac{(x(t+T)-x(t))}{T}, & T \neq 0,\end{cases}
$$


where $T$ is a sampling period. When $T \rightarrow 0$, the delta operator model will approach the continuous system before discretization and reflect a quasicontinuous performance.

The stability of positive switched systems has been concerned by many researchers [27-29]. Most of them are based on the concept of Lyapunov stability, which is defined over an infinite-time interval. In practice, however, not only the system stability (generally refers to Lyapunov stability) but also the state trajectory over a fixed short time is needed to be considered. The finite-time stability, which is different from Lyapunov stability, admits that the state does not exceed a certain bound during a fixed finite-time interval. It should be noted that a finite-time stable system may not be stable in the sense of Lyapunov, and a Lyapunov stable system may not be finite-time stable since its transient response may exceed the bound [30]. Some early results on finite-time stability can be found in [31-33]. And recently, many results on finitetime control for switched systems have been presented in the literature [34-36]. The definition of finite-time stability for positive switched systems has been given in [37, 38]. In addition, exogenous disturbances are very common in many kinds of engineering systems, and the outputs will be inevitably affected by the disturbances. It is because of the peculiar nonnegative property of positive systems, the $l_{1}$ gain index $[39,40]$ can characterize the disturbance rejection property. Then we can limit the effect of disturbance in a prescribed level by means of $l_{1}$-gain. Moreover, it should be noted that delays are universal in practice and their existence may give rise to the deterioration of system performance and instability [41-44]. To the best of our knowledge, few results on the issues of finite-time stability and $l_{1}$-gain finite-time boundedness of positive switched systems with time-varying delay via delta operator approach have been proposed to date, which motivates the present research.

In this paper, we focus our attention on investigating the problem of finite-time $l_{1}$-gain control for a class of positive switched systems with time-varying delay via delta operator approach. The main contributions of this paper are threefold: (1) definitions of finite-time boundedness and $l_{1}$ gain performance are for the first time extended to positive switched delta operator systems with time-varying delay; (2) by applying the average dwell time approach, sufficient conditions for the existence of finite-time boundedness of the underlying system are given. Moreover, the results obtained can degenerate into the corresponding ones both in continuous-time systems and discrete-time systems; (3) a state feedback controller design scheme is proposed to guarantee that the closed-loop system is finite-time bounded with $l_{1}$-gain performance.

The remainder of the paper is organized as follows. The problem formulation and some necessary lemmas are provided in Section 2. In Section 3, $l_{1}$-gain finite-time boundedness analysis and controller design are developed. A numerical example is presented to demonstrate the feasibility of the obtained results in Section 4. Section 5 concludes this paper.

Notations. $A \succeq 0(\preceq, \succ, \prec)$ means that all entries of matrix $A$ are nonnegative (nonpositive, positive, and negative); $A>$
$B(A \geq B)$ means that $A-B>0(A-B \geq 0) ; A^{T}$ means the transpose of matrix $A ; R\left(R_{+}\right)$is the set of all real (positive real) numbers; $R^{n}$ is the $n$-dimensional real vector space; $R_{+}^{n}$ is the set of all $n$-dimensional positive real vectors; $R^{m \times n}$ is the set of all $m \times n$-dimensional real matrices. The vector 1-norm is denoted by $\|x\|=\sum_{k=1}^{n}\left|x_{k}\right|$, where $x_{k}$ is the $k$ th element of $x \in R^{n}$. Given $v: R \rightarrow R^{n}$, the $l_{1}$-norm is defined by $\|v\|_{L_{1}}=\sum_{k_{0}}^{\infty}\|v(k)\| ; 1_{n} \in R^{n}$ denotes a column vector with $n$ rows containing only 1 entry; $l_{1}\left[k_{0}, \infty\right)$ is the space of absolute integrable vector-valued functions on $\left[k_{0}, \infty\right)$; that is, we say $z:\left[k_{0}, \infty\right) \rightarrow R^{k}$ is in $l_{1}\left[k_{0}, \infty\right)$ if $\sum_{k_{0}}^{\infty}\|z(k)\|<0$.

\section{Problem Formulation}

Consider the following switched delta operator system with time-varying delay:

$$
\begin{gathered}
\delta x(k)=A_{\sigma(k)} x(k)+A_{d \sigma(k)} x\left(k-d_{k}\right)+D_{\sigma(k)} w(k), \\
x(k)=\varphi(k), \quad k=-\bar{d},-\bar{d}+1, \ldots, 0, \\
z(k)=C_{\sigma(k)} x(k)+E_{\sigma(k)} w(k),
\end{gathered}
$$

where $x(k) \in R^{n}$ denotes the state; $z(k) \in R^{z}$ is the controlled output; $k$ represents the time $t=k T ; T>0$ is the sampling period. $w(k) \in R^{w}$ is the disturbance input satisfying

$$
\sum_{k=0}^{T_{f}}\|w(k)\|<d, \quad d \geq 0,
$$

and $\sigma(k):[0, \infty) \rightarrow \underline{m}=\{1,2, \ldots, m\}$ is the switching signal with $m$ representing the number of subsystems. $A_{p}$, $A_{d p}, C_{p}, D_{p}$, and $E_{p}$ are constant matrices with appropriate dimensions. $d_{k}$ denotes the time-varying discrete delay which satisfies $0 \leq \underline{d} \leq d_{k} \leq \bar{d}$ for known constants $\underline{d}$ and $\bar{d}$; $\{\varphi(k), k=-\bar{d},-\bar{d}+1, \ldots, 0\}$ is a given discrete vector-valued initial condition. The switch is assumed to only occur at the sampling time in this paper.

Remark 1. Since a delta operator system can be regarded as a quasicontinuous system when $T \rightarrow 0$, the term $\delta x(k)$ can be applicable in normal continuous-time systems.

Definition 2. System (2) is said to be positive if, for any initial conditions $\varphi(k) \geq 0, k=-\bar{d},-\bar{d}+1, \ldots, 0$, any inputs $w(k) \succeq$ 0 , and any switching signals $\sigma(k)$, the system satisfies $x(k) \geq$ 0 and $z(k) \geq 0$ for all $k \geq 0$.

Lemma 3. System (2) is positive if and only if $\left(I+T A_{p}\right) \geq 0$, $A_{d p} \geq 0, D_{p} \geq 0, C_{p} \geq 0$, and $E_{p} \geq 0$, for all $p \in \underline{m}$.

Proof. From the definition of delta operator $\delta$, the discrete form of system (2) can be obtained as follows:

$$
\begin{aligned}
& x(k+1)=\left(I+T A_{\sigma(k)}\right) x(k) \\
&+T A_{d \sigma(k)} x\left(k-d_{k}\right)+T D_{\sigma(k)} w(k), \\
& x(k)= \varphi(k), \quad k=-\bar{d},-\bar{d}+1, \ldots, 0, \\
& z(k)=C_{\sigma(k)} x(k)+E_{\sigma(k)} w(k),
\end{aligned}
$$


Combining Lemma 2 in [45] and Lemma 1 in [28], one can obtain the remaining proof easily.

Remark 4. When $T \rightarrow 0$, system (2) degenerates to a general continuous-time positive switched system as follows:

$$
\begin{gathered}
\dot{x}(t)=A_{\sigma(t)} x(t)+A_{d \sigma(t)} x(t-d(t))+D_{\sigma(t)} w(t), \\
x(t)=\varphi(t), \quad t \in[-\bar{d}, 0] \\
z(t)=C_{\sigma(t)} x(t)+E_{\sigma(t)} w(t),
\end{gathered}
$$

where $d(t)$ denotes the time-varying delay which is everywhere time differentiable and satisfies $0 \leq \underline{d} \leq d(t) \leq \bar{d}$, and $\dot{d}(t) \leq d_{d}<1$ for known constants $\underline{d}$, $\bar{d}$, and $d_{d}$. Then, according to [39], system (5) is positive if and only if $A_{p}$ are Metzler matrices, and $A_{d p} \geq 0, C_{p} \geq 0, D_{p} \geq 0$, and $E_{p} \succeq 0$, for all $p \in \underline{m}$.

Definition 5 (see [46]). For any switching signal $\sigma(k)$ and any $k_{2}>k_{1} \geq 0$, let $N_{\sigma}\left(k_{1}, k_{2}\right)$ denote the number of switches of $\sigma(k)$ over the interval $\left[k_{1}, k_{2}\right)$. For given $\tau_{a}>0$ and $N_{0} \geq 0$, if the inequality

$$
N_{\sigma}\left(k_{1}, k_{2}\right) \leq N_{0}+\frac{k_{2}-k_{1}}{\tau_{a}}
$$

holds, then the positive constant $\tau_{a}$ is called the average dwell time and $N_{0}$ is called the chattering bound.

Without lose of generality, we choose $N_{0}=0$ in this paper.

In order to obtain the main results, we need to give the definitions of finite-time stability, finite-time boundedness and $l_{1}$-gain finite-time boundedness for positive system (2).

Definition 6 (finite-time stability). For a given time constant $T_{f}$ and two vectors $\varsigma>\varepsilon>0$, positive switched delta operator system (2) with $w(k)=0$ is said to be finite-time stable with respect to $\left(\varsigma, \varepsilon, T_{f}, \sigma(k)\right)$, if $\sup _{-\bar{d} \leq k \leq 0}\left\{x^{T}(k) \varsigma\right\} \leq$ $1 \Rightarrow x^{T}(k) \varepsilon<1$, for all $k \in\left[0, T_{f}\right)$. If the above condition is satisfied for any switching signals $\sigma(k)$, positive system (2) is said to be uniformly finite-time stable with respect to $\left(\varsigma, \varepsilon, T_{f}\right)$.

Remark 7. As can be seen from Definition 6, the concept of finite-time stability is different from the one of Lyapunov asymptotic stability. A Lyapunov asymptotically stable switched system may not be finite-time stable because its states may exceed the prescribed bounds during the interval time.

Definition 8 (finite-time boundedness). For a given time constant $T_{f}$ and two vectors $\varsigma>\varepsilon>0$, positive switched delta operator system (2) is said to be finite-time bounded with respect to $\left(\varsigma, \varepsilon, T_{f}, d, \sigma(k)\right)$, if $\sup _{-\bar{d} \leq k \leq 0}\left\{x^{T}(k) \varsigma\right\} \leq 1 \Rightarrow$ $x^{T}(k) \varepsilon<1$, for all $k \in\left[0, T_{f}\right)$.

Definition 9 ( $l_{1}$-gain finite-time boundedness). For a given time constant $T_{f}$ and two vectors $\varsigma>\varepsilon>0$, positive switched delta operator system (2) is said to be $l_{1}$-gain finite-time bounded with respect to $\left(\varsigma, \varepsilon, T_{f}, d, \sigma(k)\right)$, if the following conditions are satisfied.

(1) Positive switched delta operator system (2) is finitetime bounded with respect to $\left(\varsigma, \varepsilon, T_{f}, d, \sigma(k)\right)$.

(2) Under zero-initial condition, that is, $\varphi(k)=0, k=$ $-\bar{d},-\bar{d}+1, \ldots, 0$, the output $z(k)$ satisfies

$$
\sum_{k=0}^{T_{f}}(1+T \rho)^{-k}\|z(k)\| \leq \gamma \sum_{k=0}^{T_{f}}\|w(k)\|,
$$

where $\rho>0$ and $\gamma>0$ are constants.

Remark 10. In Definition 9, as proposed in [39], $l_{1}$-gain performance characterizes system's suppression to exogenous disturbance.

The aim of this paper is to find a class of switching signals $\sigma(k)$ and determine a state feedback controller $u(k)=$ $K_{\sigma(k)} x(k)$ for the following positive switched delta operator system with time-varying delay:

$$
\begin{gathered}
\delta x(k)=A_{\sigma(k)} x(k)+A_{d \sigma(k)} x\left(k-d_{k}\right) \\
+B_{\sigma(k)} u(k)+D_{\sigma(k)} w(k), \\
x(k)=\varphi(k), \quad k=-\bar{d},-\bar{d}+1, \ldots, 0, \\
z(k)=C_{\sigma(k)} x(k)+E_{\sigma(k)} w(k)
\end{gathered}
$$

such that the resulting closed-loop system is positive and finite-time bounded with $l_{1}$-gain performance.

\section{Main Results}

3.1. Finite-Time Stability and Boundedness Analysis. This section will focus on the problem of finite-time boundedness for positive switched delta operator system (2).

Theorem 11. Consider positive system (2); for a given positive constant $\alpha>0$, a sampling period $T$, a time constant $T_{f}$, and two vectors $\varsigma>\varepsilon>0$, if there exist positive vectors $\nu_{p}$ and $v_{p}$, for all $p \in \underline{m}$, and positive constants $\xi_{1}, \xi_{2}$, and $\xi_{3}$, such that the following inequalities hold:

$$
\begin{gathered}
A_{p}^{T} v_{p}-\alpha v_{p}+(1+T \alpha)(\bar{d}-\underline{d}+1) v_{p} \preceq 0 \\
A_{d p}^{T} v_{p}-(1+T \alpha)^{\bar{d}+1} v_{p} \preceq 0 \\
\xi_{1} \varepsilon \prec v_{p} \prec \xi_{2} \varsigma, \quad v_{p} \prec \xi_{3} \varsigma \\
(1+T \alpha)^{T_{f}}\left(\xi_{2}+\xi_{3} T \bar{d}(1+T \alpha)^{\bar{d}}(\bar{d}-\underline{d}+1)+T d \omega\right)<\xi_{1},
\end{gathered}
$$

where $v_{p}=\left[v_{p 1}, v_{p 2}, \ldots, v_{p n}\right]^{T}$ and $v_{p}=\left[v_{p 1}, v_{p 2}, \ldots, v_{p n}\right]^{T}$, then the system is finite-time bounded with respect to 
$\left(\varsigma, \varepsilon, T_{f}, d, \sigma(k)\right)$ under the following average dwell time scheme:

$$
\begin{aligned}
\tau_{a} & >\tau_{a}^{*} \\
& =\frac{T_{f} \ln \mu}{\ln \xi_{1}-\ln \left[(1+T \alpha)^{T_{f}}\left(\xi_{2}+\xi_{3} T \bar{d}(1+T \alpha)^{\bar{d}}(\bar{d}-\underline{d}+1)+T d \omega\right)\right]},
\end{aligned}
$$

where $\omega=\max _{(r, p) \in \underline{w} \times \underline{m}}\left(\omega_{p r}\right), \underline{w}=\{1,2, \ldots, w\}, \omega_{p r}$ is the rth element of the vector $\omega_{p}=\xi_{2} D_{p}^{T} \varsigma$, and $\mu \geq 1$ satisfies

$$
v_{p} \preceq \mu v_{q}, \quad v_{p} \preceq \mu v_{q}, \quad \forall p, q \in \underline{m} .
$$

Proof. Choose the following piecewise copositive type Lyapunov functional for positive system (2):

$$
V(k, x(k))=V_{\sigma(k)}(k, x(k))
$$

The form of each $V_{p}(k, x(k))(\forall p \in \underline{m})$ is given by

$$
\begin{aligned}
V_{p}(k, x(k))= & V_{p 1}(k, x(k)) \\
& +V_{p 2}(k, x(k))+V_{p 3}(k, x(k)),
\end{aligned}
$$

where

$$
\begin{gathered}
V_{p 1}(k, x(k))=x^{T}(k) v_{p}, \\
V_{p 2}(k, x(k))=T \sum_{s=k-d_{k}}^{k-1}(1+T \alpha)^{k-s} x^{T}(s) v_{p}, \\
V_{p 3}(k, x(k))=T \sum_{l=-\bar{d}+1}^{-d} \sum_{s=k+l}^{k-1}(1+T \alpha)^{k-s} x^{T}(s) v_{p}, \quad \forall p \in \underline{m} .
\end{gathered}
$$

For simplicity, $V_{p}(k, x(k))$ is written as $V_{p}(k)$ (correspondingly, $V(k, x(k))$ is written as $V(k))$ in the later section of the paper.

Along the state decay of positive system (2), the Lyapunov function in delta domain has the following form:

$$
\begin{aligned}
& \delta V_{p 1}(k, x(k)) \\
& \quad=\delta\left(x^{T}(k) v_{p}\right)=\left(\delta x^{T}(k)\right) v_{p} \\
& \quad=x^{T}(k) A_{p}^{T} \nu_{p}+x^{T}\left(k-d_{k}\right) A_{d p}^{T} \nu_{p}+w^{T}(k) D_{p}^{T} v_{p} \\
& \delta V_{p 2}(k, x(k)) \\
& =\frac{1}{T}\left[V_{p 2}(k+1)-V_{p 2}(k)\right]
\end{aligned}
$$

$$
\begin{aligned}
= & \frac{1}{T}\left[T \sum_{s=k+1-d_{k+1}}^{(k+1)-1}(1+T \alpha)^{k+1-s} x^{T}(s) v_{p}\right. \\
& \left.-T \sum_{s=k-d_{k}}^{k-1}(1+T \alpha)^{k-s} x^{T}(s) v_{p}\right] \\
\leq & T \alpha \sum_{s=k-d_{k}}^{k-1}(1+T \alpha)^{k-s} x^{T}(s) v_{p} \\
& +(1+T \alpha) x^{T}(k) v_{p}-(1+T \alpha)^{\bar{d}+1} x^{T}\left(k-d_{k}\right) v_{p} \\
& +\sum_{s=k+1-\bar{d}}^{k-d}(1+T \alpha)^{k+1-s} x^{T}(s) v_{p}
\end{aligned}
$$$$
\delta V_{p 3}(k, x(k))
$$$$
=\frac{1}{T}\left[V_{p 3}(k+1)-V_{p 3}(k)\right]
$$$$
=\frac{1}{T}\left[T \sum_{l=-\bar{d}+1}^{-\frac{d}{T}} \sum_{s=k+1+l}^{(k+1)-1}(1+T \alpha)^{k+1-s} x^{T}(s) v_{p}\right.
$$$$
\left.-T \sum_{l=-\bar{d}+1}^{-\underline{d}} \sum_{s=k+l}^{k-1}(1+T \alpha)^{k-s} x^{T}(s) v_{p}\right]
$$$$
=T \alpha \sum_{l=-\bar{d}+1}^{-\underline{d}} \sum_{s=k+l}^{k-1}(1+T \alpha)^{k-s} x^{T}(s) v_{p}
$$$$
+(1+T \alpha)(\bar{d}-\underline{d}) x^{T}(k) v_{p}
$$$$
-\sum_{s=k+1-\bar{d}}^{k-d}(1+T \alpha)^{k+1-s} x^{T}(s) v_{p} .
$$

According to (18), we have

$$
\begin{aligned}
\delta V_{p}(k, x(k))-\alpha V_{p}(k, x(k)) & \\
\leq & x^{T}(k) A_{p}^{T} v_{p}+x^{T}\left(k-d_{k}\right) A_{d p}^{T} v_{p} \\
& +w^{T}(k) D_{p}^{T} v_{p}-\alpha x^{T}(k) v_{p} \\
& +(1+T \alpha) x^{T}(k) v_{p}-(1+T \alpha)^{\bar{d}+1} x^{T}\left(k-d_{k}\right) v_{p} \\
& +\sum_{s=k+1-\bar{d}}^{k-d}(1+T \alpha)^{k+1-s} x^{T}(s) v_{p} \\
& +(1+T \alpha)(\bar{d}-\underline{d}) x^{T}(k) v_{p} \\
& -\sum_{s=k+1-\bar{d}}^{k-d}(1+T \alpha)^{k+1-s} x^{T}(s) v_{p}
\end{aligned}
$$




$$
\begin{aligned}
\leq & x^{T}(k)\left[A_{p}^{T} v_{p}-\alpha v_{p}+(1+T \alpha)(\bar{d}-\underline{d}+1) v_{p}\right] \\
& +x^{T}\left(k-d_{k}\right)\left[A_{d p}^{T} v_{p}-(1+T \alpha)^{\bar{d}+1} v_{p}\right]+w^{T}(k) D_{p}^{T} v_{p} .
\end{aligned}
$$

According to (9)-(11), we can easily obtain

$$
\delta V_{p}(k)-\alpha V_{p}(k) \leq w^{T}(k) D_{p}^{T} v_{p} \leq w^{T}(k) D_{p}^{T} \zeta \xi_{2} .
$$

Denoting $\omega_{p}=D_{p}^{T} \varsigma \xi_{2}$, it follows from (20) that

$$
\begin{aligned}
\delta V_{p}(k) & -\alpha V_{p}(k) \leq w^{T}(k) \omega_{p} \\
& \Longrightarrow \delta V_{p}(k)=\frac{V_{p}(k+1)-V_{p}(k)}{T} \\
& \leq \alpha V_{p}(k)+w^{T}(k) \omega_{p} \\
& \Longrightarrow V_{p}(k+1) \leq V_{p}(k)+T \alpha V_{p}(k)+T w^{T}(k) \omega_{p} \\
& \Longrightarrow V_{p}(k+1) \leq(1+T \alpha) V_{p}(k)+T w^{T}(k) \omega_{p} .
\end{aligned}
$$

Then, along the trajectory of positive system (2), for $k \in$ $\left[k_{i}, k_{i+1}\right)$,

$$
\begin{aligned}
V_{\sigma(k)}(k) \leq & (1+T \alpha)^{k-k_{i}} V_{\sigma\left(k_{i}\right)}\left(k_{i}\right) \\
& +\sum_{s=k_{i}}^{k-1}(1+T \alpha)^{k-s-1} T w^{T}(s) \emptyset_{p} .
\end{aligned}
$$

From (14) and (16), we can obtain

$$
V_{\sigma\left(k_{i}\right)}\left(k_{i}\right) \leq \mu V_{\sigma\left(k_{i}^{-}\right)}\left(k_{i}^{-}\right) .
$$

Let $M$ be the switching number of $\sigma(k)$ over $\left[0, T_{f}\right)$, and denote $k_{1}, k_{2}, k_{3}, \ldots, k_{M}$ as the switching instants over the interval $\left[0, T_{f}\right)$. Then, for $k \in\left[0, T_{f}\right)$, we obtain from (22), and (23) and the relation $N_{\sigma(k)}(0, k) \leq k / \tau_{a}$ that

$$
\begin{aligned}
V(k) \leq & (1+T \alpha)^{\left(k-k_{M}\right)} V_{\sigma\left(k_{M}\right)}\left(k_{M}\right) \\
& +\sum_{s=k_{M}}^{k-1}(1+T \alpha)^{(k-s-1)} T w^{T}(s) \omega_{\sigma\left(k_{M}\right)} \\
\leq & \mu(1+T \alpha)^{\left(k-k_{M}\right)} V_{\sigma\left(k_{M}^{-}\right)}\left(k_{M}^{-}\right) \\
& +\sum_{s=k_{M}}^{k-1}(1+T \alpha)^{(k-s-1)} T w^{T}(s) \omega_{\sigma\left(k_{M}\right)} \\
\leq & \mu(1+T \alpha)^{\left(k-k_{M-1}\right)} V_{\sigma\left(k_{M-1}\right)}\left(k_{M-1}\right) \\
& +\mu \sum_{s=k_{M-1}}^{k_{M}-1}(1+T \alpha)^{(k-s-1)} T w^{T}(s) \omega_{\sigma\left(k_{M-1}\right)} \\
& +\sum_{s=k_{M}}^{k-1}(1+T \alpha)^{(k-s-1)} T w^{T}(s) \omega_{\sigma\left(k_{M}\right)}
\end{aligned}
$$

$\leq \cdots$

$\leq \mu^{M}(1+T \alpha)^{k} V_{\sigma(0)}(0)$

$+\mu^{M} \sum_{s=0}^{k_{1}-1}(1+T \alpha)^{(k-s-1)} T w^{T}(s) \omega_{\sigma(0)}$ $+\mu^{M-1} \sum_{s=k_{1}}^{k_{2}-1}(1+T \alpha)^{(k-s-1)} T w^{T}(s){\omega_{\sigma\left(k_{1}\right)}}$

$+\cdots+\sum_{s=k_{M}}^{k-1}(1+T \alpha)^{(k-s-1)} T w^{T}(s) \omega_{\sigma\left(k_{M}\right)}$

$=\mu^{M}(1+T \alpha)^{T_{f}} V_{\sigma(0)}(0)$

$+\sum_{s=0}^{k-1} \mu^{N_{\sigma(k)}(s, k)}(1+T \alpha)^{(k-s-1)} T w^{T}(s) \omega_{\sigma(s)}$

$\leq \mu^{M}(1+T \alpha)^{T_{f}} V_{\sigma(0)}(0)$

$+\mu^{M}(1+T \alpha)^{T_{f}} \sum_{s=0}^{k-1} T w^{T}(s) \omega_{\sigma(s)}$

$\leq \mu^{M}(1+T \alpha)^{T_{f}}\left(V_{\sigma(0)}(0)+T d \omega\right)$.

Considering the definition of $V_{\sigma(k)}(k)$, it yields that

$$
V_{\sigma(k)}(k) \geq \xi_{1} x^{T}(k) \varepsilon,
$$

$$
\begin{aligned}
& V_{\sigma(0)}(0) \\
& \leq \xi_{2} x^{T}(0) \varsigma+\xi_{3} T \bar{d}(1+T \alpha)^{\bar{d}} \\
& \quad \times\left[\sup _{-\bar{d} \leq \theta \leq-1}\left\{x^{T}(\theta) \varsigma\right\}+(\bar{d}-\underline{d}) \sup _{-\bar{d}+1 \leq \theta \leq-1}\left\{x^{T}(\theta) \varsigma\right\}\right] \\
& \leq\left[\xi_{2}+\xi_{3} T \bar{d}(1+T \alpha)^{\bar{d}}(\bar{d}-\underline{d}+1)\right] \sup _{-\bar{d} \leq \theta \leq 0}\left\{x^{T}(\theta) \varsigma\right\} \\
& \leq\left[\xi_{2}+\xi_{3} T \bar{d}(1+T \alpha)^{\bar{d}}(\bar{d}-\underline{d}+1)\right] .
\end{aligned}
$$

Combining (24)-(25), we obtain

$$
\begin{aligned}
x^{T}(k) \varepsilon \leq & \frac{1}{\xi_{1}}\left[\mu^{1 / \tau_{a}}(1+T \alpha)\right]^{T_{f}} \\
& \times\left(\xi_{2}+\xi_{3} T \bar{d}(1+T \alpha)^{\bar{d}}(\bar{d}-\underline{d}+1)+T d \omega\right) .
\end{aligned}
$$

Substituting (13) into (26) leads to

$$
x^{T}(k) \varepsilon<1 .
$$


According to Definition 8, we can conclude that the positive switched delta operator system (2) is finite-time bounded with respect to $\left(\varsigma, \varepsilon, T_{f}, d, \sigma(k)\right)$.

The proof is completed.

Remark 12. It should be noted that there is no requirement of negative definitiveness on $\delta V(k)$, which is different from the case of classical Lyapunov stability.

Remark 13. When $\mu=1$ in (14), which leads to $\nu_{p}=v_{q}, v_{p}=$ $v_{q}$, for all $p, q \in \underline{m}$, and $\tau_{a}^{*}=0$ by (13), system (2) possesses a common co-positive type Lyapunov-Krasovskii functional, and the switching signal can be arbitrary.

When $w(k)=0$, the result on finite-time stability can be obtained as follows.

Corollary 14. Consider positive system (2) with $w(k)=0$; for a given positive constant $\alpha>0$, a sampling period T, a time constant $T_{f}$, and two vectors $\varsigma>\varepsilon>0$, if there exist positive vectors $v_{p}$ and $v_{p}$, for all $p \in \underline{m}$, and positive constants $\xi_{1}, \xi_{2}$, and $\xi_{3}$, such that (9)-(11) and the following inequality hold:

$$
(1+T \alpha)^{T_{f}}\left(\xi_{2}+\xi_{3} T \bar{d}(1+T \alpha)^{\bar{d}}(\bar{d}-\underline{d}+1)\right)<\xi_{1},
$$

then, the system is finite-time stable with respect to $\left(\varsigma, \varepsilon, T_{f}, \sigma(k)\right)$ under the following average dwell time scheme

$$
\begin{aligned}
\tau_{a} & >\tau_{a}^{*} \\
& =\frac{T_{f} \ln \mu}{\ln \xi_{1}-\ln \left[(1+T \alpha)^{T_{f}}\left(\xi_{2}+\xi_{3} T \bar{d}(1+T \alpha)^{\bar{d}}(\bar{d}-\underline{d}+1)\right)\right]},
\end{aligned}
$$

where $\mu \geq 1$ satisfies (14).

Remark 15. As stated in [38], the general idea of finite-time stability concerns the boundedness of the state of a system over a finite interval for given initial conditions. On the other hand, the idea of finite-time boundedness concerns the behaviors of the state in the presence of both given initial conditions and external disturbances. It is easy to see from Definitions 6 and 8 that finite-time stability can be regarded as a special case by setting $d=0$.

Remark 16. When the sampling period $T \rightarrow 0$, positive system (2) becomes a continuous-time positive system as (5). In this case, Theorem 11 will generate to Theorem 1 proposed in [38].

Let $\bar{A}_{\sigma(k)}=A_{\sigma(k)}+I$. When the sampling period $T=1$, positive system (2) becomes a discrete-time positive system as follows:

$$
\begin{gathered}
x(k+1)=\bar{A}_{\sigma(k)} x(k)+A_{d \sigma(k)} x\left(k-d_{k}\right) \\
+D_{\sigma(k)} w(k), \\
x(k)=\varphi(k), \quad k=-\bar{d},-\bar{d}+1, \ldots, 0, \\
z(k)=C_{\sigma(k)} x(k)+E_{\sigma(k)} w(k),
\end{gathered}
$$

where $\bar{A}_{p} \geq 0, A_{d p} \geq 0, C_{p} \geq 0$, and $E_{p} \geq 0$, for all $p \in \underline{m}$. Then we have the following result.

Corollary 17. Consider positive system (30); for a given positive constant $\alpha>0$, a time constant $T_{f}$, and two vectors $\varsigma>\varepsilon>0$, if there exist positive vectors $\nu_{p}$ and $v_{p}$, for all $p \in \underline{m}$, and positive constants $\xi_{1}, \xi_{2}$, and $\xi_{3}$, such that (11) and the following inequalities hold:

$$
\begin{gathered}
\bar{A}_{p}^{T} v_{p}-\alpha v_{p}+(1+\alpha)(\bar{d}-\underline{d}+1) v_{p} \preceq 0, \\
A_{d p}^{T} \nu_{p}-(1+\alpha)^{\bar{d}+1} v_{p} \preceq 0, \\
(1+\alpha)^{T_{f}}\left(\xi_{2}+\xi_{3} \bar{d}(1+\alpha)^{\bar{d}}(\bar{d}-\underline{d}+1)+d \omega\right)<\xi_{1},
\end{gathered}
$$

then the system is finite-time bounded with respect to $\left(\varsigma, \varepsilon, T_{f}, d, \sigma(k)\right)$ under the following average dwell time scheme:

$$
\begin{aligned}
\tau_{a} & >\tau_{a}^{*} \\
& =\frac{T_{f} \ln \mu}{\ln \xi_{1}-\ln \left[(1+\alpha)^{T_{f}}\left(\xi_{2}+\xi_{3} \bar{d}(1+\alpha)^{\bar{d}}(\bar{d}-\underline{d}+1)+d \omega\right)\right]},
\end{aligned}
$$

where $\omega$ has been defined in Theorem 11 and $\mu \geq 1$ satisfies (14).

3.2. $l_{1}$-Gain Finite-Time Boundedness Analysis. In this section, we will consider the problem of $l_{1}$-gain finite-time boundedness for positive system (2).

Theorem 18. Consider positive system (2); for a given positive constant $\alpha>0$, a sampling period $T$, a given time constant $T_{f}$ and two vectors $\varsigma>\varepsilon>0$, if there exist positive vectors $v_{p}$ and $v_{p}$, for all $p \in \underline{m}$, and positive constants $\gamma, \xi_{1}, \xi_{2}$ and $\xi_{3}$, such that (10), (11), and the following inequalities hold:

$$
\begin{gathered}
A_{p}^{T} \nu_{p}-\alpha v_{p}+(1+T \alpha)(\bar{d}-\underline{d}+1) v_{p}+\widetilde{c}_{p} \preceq 0, \\
D_{p}^{T} \nu_{p}+\tilde{e}_{p}-\gamma 1_{w} \preceq 0, \quad \omega<\gamma \\
(1+T \alpha)^{T_{f}}\left(\xi_{2}+\xi_{3} T \bar{d}(1+T \alpha)^{\bar{d}}(\bar{d}-\underline{d}+1)+T d \gamma\right)<\xi_{1},
\end{gathered}
$$

where $\omega$ has been defined in Theorem 11, $v_{p}=$ $\left[v_{p 1}, v_{p 2}, \ldots, v_{p n}\right]^{T}, \quad v_{p}=\left[v_{p 1}, v_{p 2}, \ldots, v_{p n}\right]^{T}, 1_{w}=$ $[\underbrace{1,1, \ldots, 1}]^{T}, \widetilde{c}_{p}=\left[\left\|c_{p 1}\right\|,\left\|c_{p 2}\right\|, \ldots,\left\|c_{p n}\right\|\right]^{T}, c_{p j}$ represents the jth column of matrix $C_{p}, j \in \underline{n}=\{1,2, \ldots, n\}$. $\tilde{e}_{p}=\left[\left\|e_{p 1}\right\|,\left\|e_{p 2}\right\|, \ldots,\left\|e_{p w}\right\|\right]^{T}, e_{p j}$ represents the $j$ th column of matrix $E_{p}$, and $j \in \underline{w}=\{1,2, \ldots, w\}$, then the system is 
$l_{1}$-gain finite-time bounded with respect to $\left(\varsigma, \varepsilon, T_{f}, d, \sigma(k)\right)$ under the following average dwell time scheme:

$$
\begin{aligned}
& \tau_{a}>\tau_{a}^{*} \\
& =\max \left\{\left(T_{f} \ln \mu\right)\right. \\
& \quad \times\left(\ln \xi_{1}\right. \\
& \quad-\ln \left[(1+T \alpha)^{T_{f}}\right. \\
& \times\left(\xi_{2}+\xi_{3} T \bar{d}(1+T \alpha)^{\bar{d}}(\bar{d}-\underline{d}+1)\right. \\
& \left.+\frac{\ln \mu}{\ln (1+T \alpha)}\right\},
\end{aligned}
$$

where $\mu \geq 1$ satisfies (14).

Proof. Equation (9) can be directly derived from (33). By Theorem 11, we can easily obtain from (10)-(11) and (33)(35) that system (2) is finite-time bounded with respect to $\left(\varsigma, \varepsilon, T_{f}, d, \sigma(k)\right)$. To show the weighted $l_{1}$-gain performance, we choose the piecewise co-positive type LyapunovKrasovskii functional (15). Following the proof line of Theorem 11, we can get from (10), (33), and (34) that

$$
\delta V_{p}(k)-\alpha V_{p}(k)+\|z(k)\|-\gamma\|w(k)\|<0 .
$$

Let $\Lambda(s)=T\|z(s)\|-T \gamma\|w(s)\|$; then, for $k \in\left[k_{i}, k_{i+1}\right)$, (37) gives rise to

$$
V(k) \leq(1+T \alpha)^{\left(k-k_{i}\right)} V_{\sigma\left(k_{i}\right)}\left(k_{i}\right)-\sum_{s=k_{i}}^{k-1}(1+T \alpha)^{(k-s-1)} \Lambda(s) .
$$

Following the proof line of $(24)$, for any $k \in\left[0, T_{f}\right)$, we can obtain

$$
\begin{aligned}
V_{\sigma(k)}(k) \leq & \mu^{N_{\sigma(k)}(0, k)}(1+T \alpha)^{k} V_{\sigma(0)}(0) \\
& -\sum_{s=0}^{k-1} \mu^{N_{\sigma(k)}(s, k)}(1+T \alpha)^{(k-s-1)} \Lambda(s) .
\end{aligned}
$$

Under the zero initial condition, we have from (39) that

$$
0 \leq-\sum_{s=0}^{k-1} \mu^{N_{\sigma(k)}(s, k)}(1+T \alpha)^{(k-s-1)} \Lambda(s) .
$$

Namely,

$$
\begin{aligned}
& \sum_{s=0}^{k-1} \mu^{N_{\sigma(k)}(s, k)}(1+T \alpha)^{(k-s-1)}\|z(s)\| \\
& \quad \leq \gamma \sum_{s=0}^{k-1} \mu^{N_{\sigma(k)}(s, k)}(1+T \alpha)^{(k-s-1)}\|w(s)\| .
\end{aligned}
$$

Multiplying both sides of (41) by $\mu^{-N_{\sigma(k)}(0, k)}$ yields

$$
\begin{aligned}
& \sum_{s=0}^{k-1} \mu^{-N_{\sigma(k)}(0, s)}(1+T \alpha)^{(k-s-1)}\|z(s)\| \\
& \quad \leq \gamma \sum_{s=0}^{k-1} \mu^{-N_{\sigma(k)}(0, s)}(1+T \alpha)^{(k-s-1)}\|w(s)\| .
\end{aligned}
$$

Noticing that $N_{\sigma(k)}(0, s) \leq s / \tau_{a}$ and $\tau_{a}>\ln \mu / \ln (1+T \alpha)$, we have

$$
\mu^{-N_{\sigma(k)}(0, s)} \geq(1+T \alpha)^{-s} .
$$

Combining (42) and (43) leads to

$$
\begin{aligned}
\sum_{s=0}^{k-1}(1+T \alpha)^{-s}(1+T \alpha)^{(k-s-1)}\|z(s)\| \\
\leq \gamma \sum_{s=0}^{k-1}(1+T \alpha)^{(k-s-1)}\|w(s)\| .
\end{aligned}
$$

Let $k-1=T_{f}$; then multiplying both sides of (44) by $(1+$ $T \alpha)^{-T_{f}}$ leads to

$$
\sum_{s=0}^{T_{f}}(1+T \alpha)^{-2 s}\|z(s)\| \leq \gamma \sum_{s=0}^{T_{f}}\|w(s)\| .
$$

Setting $(1+T \alpha)^{2}=1+T \rho$, we can obtain that positive system (2) is $l_{1}$-gain finite-time bounded with respect to $\left(\varsigma, \varepsilon, T_{f}, d, \sigma(k)\right)$.

The proof is completed.

3.3. $l_{1}$-Gain Controller Design. In this section, we are interested in designing a state feedback controller $u(k)=$ $K_{\sigma(k)} x(k)$ for positive switched delta operator system (8) such that the corresponding closed-loop system

$$
\begin{aligned}
& \delta x(k)=\left(A_{\sigma(k)}+B_{\sigma(k)} K_{\sigma(k)}\right) x(k) \\
&+A_{d \sigma(k)} x\left(k-d_{k}\right)+D_{\sigma(k)} w(k), \\
& x(k)=\varphi(k), \quad k=-\bar{d},-\bar{d}+1, \ldots, 0, \\
& z(k)=C_{\sigma(k)} x(k)+E_{\sigma(k)} w(k),
\end{aligned}
$$

is $l_{1}$-gain finite-time bounded with respect to $\left(\varsigma, \varepsilon, T_{f}, d, \sigma(k)\right)$. By Lemma 3 , in order to guarantee the positivity of system $(46), I+T\left(A_{p}+B_{p} K_{p}\right) \geq 0$, for all $p \in \underline{m}$, should be satisfied.

Theorem 19. Consider positive system (8); for a given positive constant $\alpha>0$, a sampling period $T$, a given time constant $T_{f}$, and two vectors $\varsigma>\varepsilon>0$, if there exist positive vectors $v_{p}$ and $v_{p}$, for all $p \in \underline{m}$, and positive constants $\gamma, \xi_{1}, \xi_{2}$, and $\xi_{3}$, such that (10)-(11), (34)-(35), and the following conditions are satisfied:

$$
\begin{gathered}
A_{p}^{T} v_{p}-\alpha v_{p}+g_{p}+(1+T \alpha)(\bar{d}-\underline{d}+1) v_{p}+\tilde{c}_{p} \preceq 0, \\
I+T\left(A_{p}+B_{p} K_{p}\right) \succeq 0,
\end{gathered}
$$


where $g_{p}=K_{p}^{T} B_{p}^{T} v_{p}$, then the resulting closed-loop system (46) is $l_{1}$-gain finite-time bounded with respect to $\left(\varsigma, \varepsilon, T_{f}, d, \sigma(k)\right)$ under the average dwell time scheme (36), where $\mu \geq 1$ satisfies (14).

Proof. Denote $g_{p}=K_{p}^{T} B_{p}^{T} v_{p}$. Following the proof line of Theorem 18, one can exactly obtain Theorem 19 . It is omitted here.

The proof is completed.

Based on Theorem 19, we are now in a position to present an effective algorithm for constructing the desired controller.

Algorithm 20.

Step 1. Input the matrices $A_{p}, A_{d p}, B_{p}, C_{p}, D_{p}$, and $E_{p}$.

Step 2. Choose the parameters $\alpha>0$ and $\gamma>0$. By solving (10)-(11), (34)-(35), and (47), one can obtain the solutions of $v_{p}, v_{p}, \vartheta_{p}$, and $g_{p}$.

Step 3. By the equation $g_{p}=K_{p}^{T} F_{p}^{T} v_{p}$ with the obtained $g_{p}$ and $v_{p}$, one can get the gain matrices $K_{p}$.

Step 4. Check condition (48) in Theorem 19. If it holds, go to Step 5; otherwise, adjust the parameter $\alpha$ and return to Step 2.

Step 5. Construct the feedback controller $u(k)=K_{p} x(k)$, where $K_{p}$ are the gain matrices.

\section{Numerical Example}

Consider positive switched delta operator system (2) consisting of two subsystems described by

subsystem 1:

$$
\begin{array}{cc}
A_{1}=\left[\begin{array}{cc}
-4 & 1 \\
2 & -3.5
\end{array}\right], & A_{d 1}=\left[\begin{array}{ll}
0.2 & 0.1 \\
0.1 & 0.2
\end{array}\right], \quad B_{1}=\left[\begin{array}{l}
0.2 \\
0.1
\end{array}\right], \\
C_{1}=\left[\begin{array}{ll}
0.1 & 0.2
\end{array}\right], & D_{1}=\left[\begin{array}{l}
0.1 \\
0.2
\end{array}\right],
\end{array}
$$

subsystem 2 :

$$
\begin{aligned}
& A_{2}=\left[\begin{array}{cc}
-4.5 & 2 \\
2 & -3
\end{array}\right], \quad A_{d 2}=\left[\begin{array}{ll}
0.3 & 0.2 \\
0.1 & 0.2
\end{array}\right], \quad B_{2}=\left[\begin{array}{l}
0.1 \\
0.2
\end{array}\right], \\
& C_{2}=\left[\begin{array}{ll}
0.1 & 0.1
\end{array}\right], \quad D_{2}=\left[\begin{array}{l}
0.2 \\
0.1
\end{array}\right], \quad E_{2}=[0.2] \text {. }
\end{aligned}
$$

Choosing $T=0.2, T_{f}=15, \alpha=0.4, \gamma=10, \bar{d}=2.0$, $\underline{d}=0, d=0.01, \varsigma=\left[\begin{array}{ll}3 & 6\end{array}\right]^{T}$, and $\varepsilon=\left[\begin{array}{ll}0.01 & 0.01\end{array}\right]^{T}$, then, by



FIGURE 1: Switching signal.



FIGURE 2: State response of the closed-loop system.

solving (10)-(11), (34)-(35), and (47) in Theorem 19, we can obtain the following solutions:

$$
\begin{array}{lll}
v_{1}=\left[\begin{array}{l}
17.9620 \\
20.8488
\end{array}\right], & v_{1}=\left[\begin{array}{c}
7.3656 \\
11.2371
\end{array}\right], & g_{1}=\left[\begin{array}{c}
6.6854 \\
13.3700
\end{array}\right], \\
v_{2}=\left[\begin{array}{l}
17.8294 \\
24.6985
\end{array}\right], & v_{2}=\left[\begin{array}{l}
8.3105 \\
9.9876
\end{array}\right], & g_{2}=\left[\begin{array}{l}
5.4706 \\
7.9281
\end{array}\right],
\end{array}
$$

and the state feedback gain matrices can be given by $g_{p}=$ $K_{p}^{T} B_{p}^{T} v_{p}$ as follows:

$$
K_{1}=\left[\begin{array}{ll}
1.1776 & 2.3550
\end{array}\right], \quad K_{2}=\left[\begin{array}{ll}
0.8138 & 1.1793
\end{array}\right] .
$$

Obviously, condition (48) is satisfied. From (14) and (36), we have $\mu=1.1961$ and $\tau_{a}^{*}=2.3272$. Choosing $\tau_{a}=$ 3 , the simulation results are shown in Figures $1-4$, where the initial conditions are $x(0)=\left[\begin{array}{ll}0.1 & 0.12\end{array}\right]^{T}$ and $x(\theta)=$ $\left[\begin{array}{ll}0 & 0\end{array}\right]^{T}, \theta=-2,-1,0$, and the exogenous disturbance input 


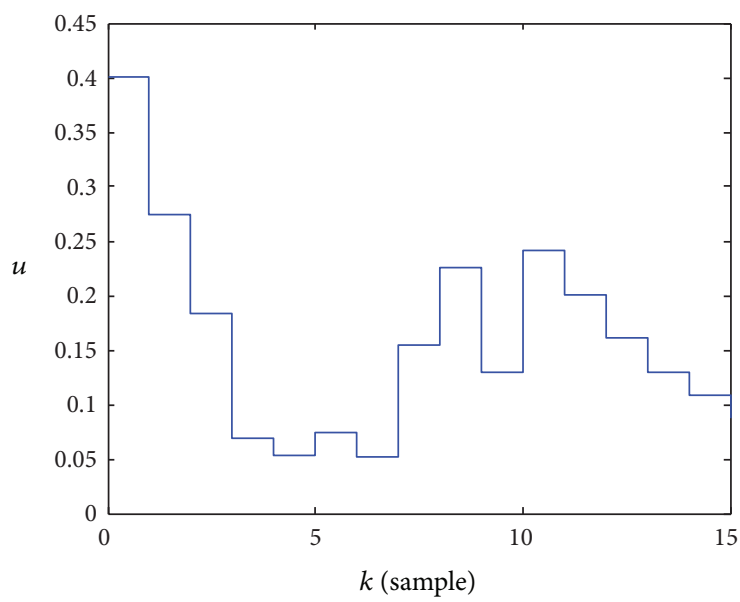

FIGURE 3: State feedback control law $u(k)=K_{\sigma(k)} x(k)$.

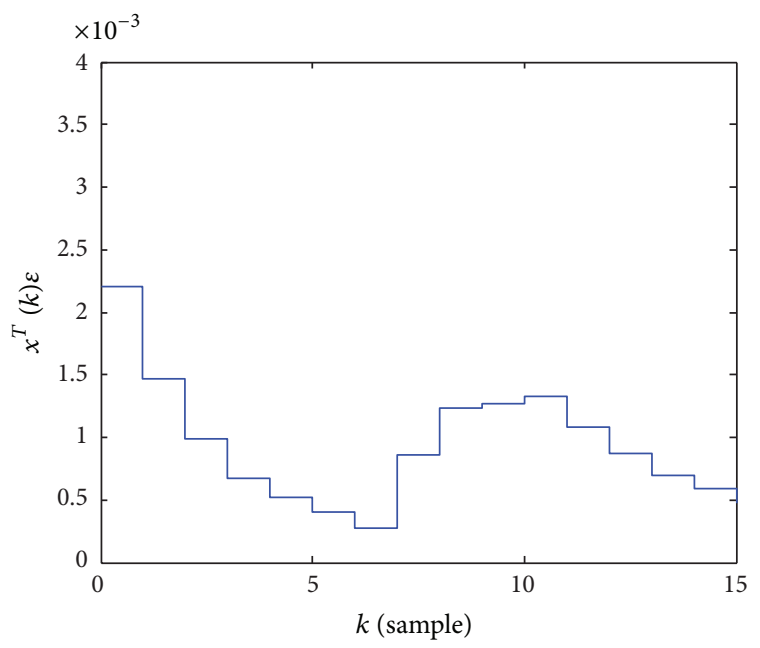

Figure 4: The evolution of $x^{T}(k) \varepsilon$.

is $w(k)=0.001 e^{-0.5 k}$ which satisfies (3). The switching signal with average dwell time $\tau_{a}=3$ is shown in Figure 1 and the state response of the closed-loop system is given in Figure 2. The corresponding control law is given in Figures 3 and 4 plots the evolution of $x^{T}(k) \varepsilon$.

It is easy to see from Figures $1-4$ that $x^{T}(k) \varepsilon<1$, for all $k \in\left[0, T_{f}\right)$, is satisfied for the given time constant $T_{f}=15$. Thus, according to Definition 9, the closed-loop system is $l_{1}$ gain finite-time bounded with respect to $\left(\varsigma, \varepsilon, T_{f}, d, \sigma(k)\right)$.

\section{Conclusions}

In this paper, finite-time boundedness and $l_{1}$-gain finite-time boundedness for a class of positive switched systems with time-varying delays via delta operator approach have been investigated. By constructing a co-positive type LyapunovKrasovskii functional and using the average dwell time approach, we have proposed sufficient conditions to ensure the finite-time stability and $l_{1}$-gain finite-time boundedness of the considered system. Based on the results obtained, a state feedback controller and a class of switching signals with the average dwell time are designed to guarantee that the corresponding closed-loop system is finite-time stable with an $l_{1}$-gain performance. Finally, a numerical example is presented to demonstrate the feasibility of the obtained results.

\section{Conflict of Interests}

The authors declare that there is no conflict of interests regarding the publication of this paper.

\section{Acknowledgment}

This work was supported by the National Natural Science Foundation of China under Grant no. 61273120.

\section{References}

[1] T. Kaczorek, Positive 1D and 2D Systems, Springer, London, UK, 2002.

[2] X. Liu, "Constrained control of positive systems with delays," IEEE Transactions on Automatic Control, vol. 54, no. 7, pp. 1596$1600,2009$.

[3] P. D. Berk, J. R. Bloomer, R. B. Howe, and N. I. Berlin, "Constitutional hepatic dysfunction (Gilbert's syndrome). A new definition based on kinetic studies with unconjugated radiobilirubin," The American Journal of Medicine, vol. 49, no. 3, pp. 296-305, 1970.

[4] E. R. Carson, C. Cobelli, and L. Finkelstein, "Modeling and identification of metabolic systems," American Journal of Physiology, vol. 240, no. 3, pp. R120-R129, 1981.

[5] F. W. Weckerly, "Matrix population models: construction, analysis and interpretation," Natural Resource Modeling, vol. 14, no. 4, pp. 593-595, 2001.

[6] R. N. Shorten, D. J. Leith, J. Foy, and R. Kilduff, "Analysis and design of AIMD congestion control algorithms in communication networks," Automatica, vol. 41, no. 4, pp. 725-730, 2005.

[7] R. Shorten, F. Wirth, and D. Leith, "A positive systems model of TCP-like congestion control: asymptotic results," IEEE/ACM Transactions on Networking, vol. 14, no. 3, pp. 616-629, 2006.

[8] L. Caccetta, L. R. Foulds, and V. G. Rumchev, "A positive linear discrete-time model of capacity planning and its controllability properties," Mathematical and Computer Modelling, vol. 40, no. 1-2, pp. 217-226, 2004.

[9] R. Shorten, D. Leith, J. Foy, and R. Kilduff, "Towards an analysis and design framework for congestion control in communication networks," in Proceedings of the 12th Yale Workshop on Adaptive and Learning Systems, New Haven, Conn, USA, May 2003.

[10] A. Jadbabaie, J. Lin, and A. Morse, "Coordination of groups of mobile autonomous agents using nearest neighbor rules," IEEE Transactions on Automatic Control, vol. 48, no. 6, pp. 988-1001, 2003.

[11] T. Kaczorek, "The choice of the forms of Lyapunov functions for a positive 2D Roesser model," International Journal of Applied Mathematics and Computer Science, vol. 17, no. 4, pp. 471-475, 2007.

[12] T. Kaczorek, "A realization problem for positive continuoustime systems with reduced numbers of delays," International 
Journal of Applied Mathematics and Computer Science, vol. 16, no. 3, pp. 325-331, 2006.

[13] L. Benvenuti, A. Santis, and L. Farina, Positive Systems, Lecture Notes in Control and Information Sciences, Springer, Berlin, Germany, 2003.

[14] E. Fornasini and M. Valcher, "Linear copositive Lyapunov functions for continuous-time positive switched systems," IEEE Transactions on Automatic Control, vol. 55, no. 8, pp. 1933-1937, 2010.

[15] L. Gurvits, R. Shorten, and O. Mason, "On the stability of switched positive linear systems," IEEE Transactions on Automatic Control, vol. 52, no. 6, pp. 1099-1103, 2007.

[16] X. Zhao, L. Zhang, P. Shi, and M. Liu, "Stability of switched positive linear systems with average dwell time switching," Automatica, vol. 48, no. 6, pp. 1132-1137, 2012.

[17] G. C. Goodwin, R. L. Leal, D. Q. Mayne, and R. H. Middleton, "Rapprochement between continuous and discrete model reference adaptive control," Automatica, vol. 22, no. 2, pp. 199-207, 1986.

[18] M. A. Garnero, G. Thomas, B. Caron, J. F. Bourgeois, and E. Irving, "Pseudo continuous identification, application to adaptive pole placement control using the delta-operator," Automatique-Productique Informatique Industrielle-Automatic Control Production Systems, vol. 26, no. 2, pp. 147-166, 1992.

[19] G. Li and M. Gevers, "Comparative study of finite wordlength effects in shift and delta operator parametrizations," in Proceedings of the 29th IEEE Conference on Decision and Control, pp. 954-959, December 1990.

[20] C. P. Neuman, "Transformation between delta and forward shift operator transfer function models," IEEE Transactions on Systems, Man and Cybernetics, vol. 23, no. 1, pp. 295-296, 1993.

[21] R. H. Middleton and G. C. Goodwin, "Improved finite word length characteristics in digital control using delta operators," IEEE Transactions on Automatic Control, vol. 31, no. 11, pp. 10151021, 1986.

[22] M. A. Hersh, "The zeros and poles of delta operator systems," International Journal of Control, vol. 57, no. 3, pp. 557-575, 1993.

[23] C. P. Neuman, "Properties of the delta operator model of dynamic physical systems," IEEE Transactions on Systems, Man and Cybernetics, vol. 23, no. 1, pp. 296-301, 1993.

[24] J. Xing, R. Wang, P. Wang, and Q. Yang, "Robust control for a class of uncertain switched time delay systems using delta operator," in Proceedings of the 12th International Conference on Control Automation Robotics \& Vision (ICARCV '12), pp. 518523, Guangzhou, China, December 2012.

[25] R. H. Wang, J. C. Xing, P. Wang, and Q. L. Yang, "Nonfragile observer design for nonlinear time delay systems using delta operator," in Proceedings of the UKACC International Conference on Control (CONTROL '12), pp. 387-393, Cardiff, UK, September 2012.

[26] Z. Zhong, G. Sun, H. R. Karimi, and J. Qiu, "Stability analysis and stabilization of T-S fuzzy delta operator systems with timevarying delay via an input-output approach," Mathematical Problems in Engineering, vol. 2013, Article ID 913234, 14 pages, 2013.

[27] X. Zhao, L. Zhang, and P. Shi, "Stability of a class of switched positive linear time-delay systems," International Journal of Robust and Nonlinear Control, vol. 23, no. 5, pp. 578-589, 2013.

[28] X. W. Liu and C. Y. Dang, "Stability analysis of positive switched linear systems with delays," IEEE Transactions on Automatic Control, vol. 56, no. 7, pp. 1684-1690, 2011.
[29] E. Fornasini and M. E. Valcher, "Stability and stabilizability of special classes of discrete-time positive switched systems," in Proceedings of the American Control Conference (ACC '11), pp. 2619-2624, San Francisco, Calif, USA, 2011.

[30] W. Xiang and J. Xiao, " $H_{\infty}$ finite-time control for switched nonlinear discrete-time systems with norm-bounded disturbance," Journal of the Franklin Institute, vol. 348, no. 2, pp. 331-352, 2011.

[31] L. Weiss and E. F. Infante, "Finite time stability under perturbing forces and on product spaces," IEEE Transactions on Automatic Control, vol. 12, no. 1, pp. 54-59, 1967.

[32] A. N. Michel and S. H. Wu, "Stability of discrete systems over a finite interval of time," International Journal of Control, vol. 9, no. 6, pp. 679-693, 1969.

[33] P. Dorato, "Short-time stability in linear time-varying systems," in Proceedings of the IRE International Convention Record Part 4, pp. 83-87, New York, NY, USA, 1961.

[34] Z. Xiang, Y.-N. Sun, and M. S. Mahmoud, "Robust finitetime $H_{\infty}$ control for a class of uncertain switched neutral systems," Communications in Nonlinear Science and Numerical Simulation, vol. 17, no. 4, pp. 1766-1778, 2012.

[35] X. Lin, H. Du, and S. Li, "Finite-time boundedness and $L_{2}$ gain analysis for switched delay systems with norm-bounded disturbance," Applied Mathematics and Computation, vol. 217, no. 12, pp. 5982-5993, 2011.

[36] H. Du, X. Lin, and S. Li, "Finite-time stability and stabilization of switched linear systems," in Proceedings of the 48th IEEE Conference on Decision and Control jointly with the 28th Chinese Control Conference, pp. 1938-1943, Shanghai, China, December 2009.

[37] G. Chen and Y. Yang, "Finite-time stability of switched positive linear systems," International Journal of Robust and Nonlinear Control, vol. 24, no. 1, pp. 179-190, 2014.

[38] M. Xiang and Z. Xiang, "Finite-time $L_{1}$ control for positive switched linear systems with time-varying delay," Communications in Nonlinear Science and Numerical Simulation, vol. 18, no. 11, pp. 3158-3166, 2013.

[39] M. Xiang and Z. Xiang, "Stability, $L_{1}$-gain and control synthesis for positive switched systems with time-varying delay," Nonlinear Analysis: Hybrid Systems, vol. 9, no. 1, pp. 9-17, 2013.

[40] C. Briat, "Robust stability analysis of uncertain linear positive system via integral linear constraints: $L_{1}$ - and $L_{\infty}$-gain characterizations," in Proceedings of the 50th IEEE Conference on Decision and Control and European Control Conference, Orlando, Fla, USA, December 2011.

[41] H. Zhang, Y. Shi, and A. Saadat Mehr, "Robust energy-to-peak filtering for networked systems with time-varying delays and randomly missing data," IET Control Theory and Applications, vol. 4, no. 12, pp. 2921-2936, 2010.

[42] H. Zhang, Y. Shi, A. Saadat Mehr, and H. Huang, "Robust FIR equalization for time-varying communication channels with intermittent observations via an LMI approach," Signal Processing, vol. 91, no. 7, pp. 1651-1658, 2011.

[43] H. Zhang and Y. Shi, "Observer-based $H_{\infty}$ feedback control for arbitrarily time-varying discrete-time systems with intermittent measurements and input constraints," Journal of Dynamic Systems, Measurement, and Control, vol. 134, no. 6, Article ID 061008, 12 pages, 2012.

[44] H. Zhang, Y. Shi, and M. Liu, " $H_{\infty}$ step tracking control for networked discrete-time nonlinear systems with integral and predictive actions," IEEE Transactions on Industrial Informatics, vol. 9, no. 1, pp. 337-345, 2012. 
[45] J. Zhang, Z. Han, and J. Huang, "Robust stabilization of discretetime positive switched systems with uncertainties and average dwell time switching," Circuits, Systems, and Signal Processing, 2013.

[46] J. P. Hespanha and A. S. Morse, "Stability of switched systems with average dwell-time," in Proceedings of the 38th IEEE Conference on Decision and Control (CDC '99), vol. 3, pp. 26552660, Phoenix, Ariz, USA, December 1999. 


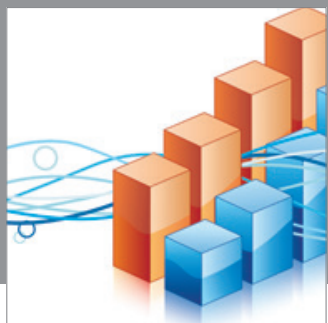

Advances in

Operations Research

mansans



The Scientific World Journal

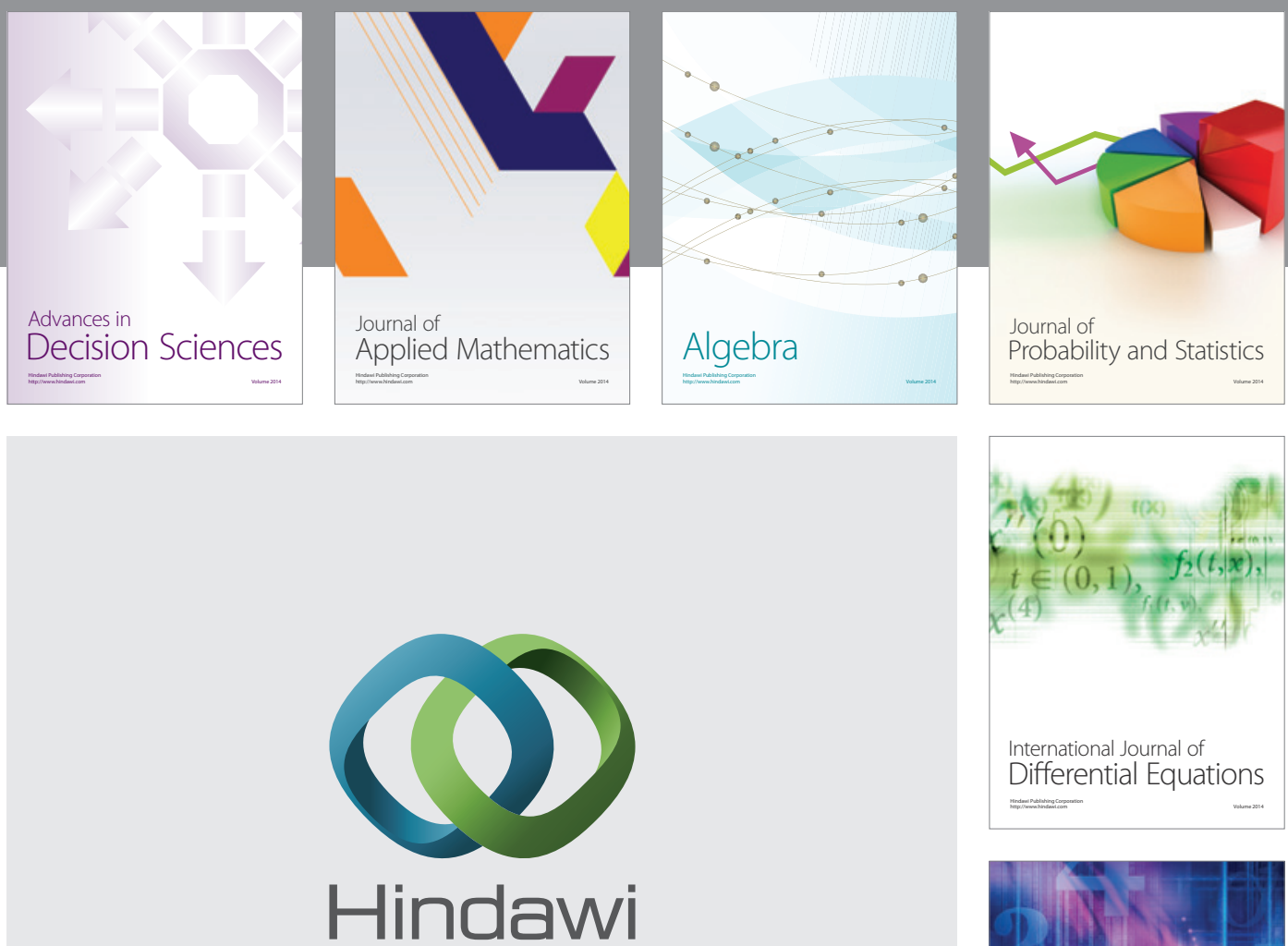

Submit your manuscripts at http://www.hindawi.com
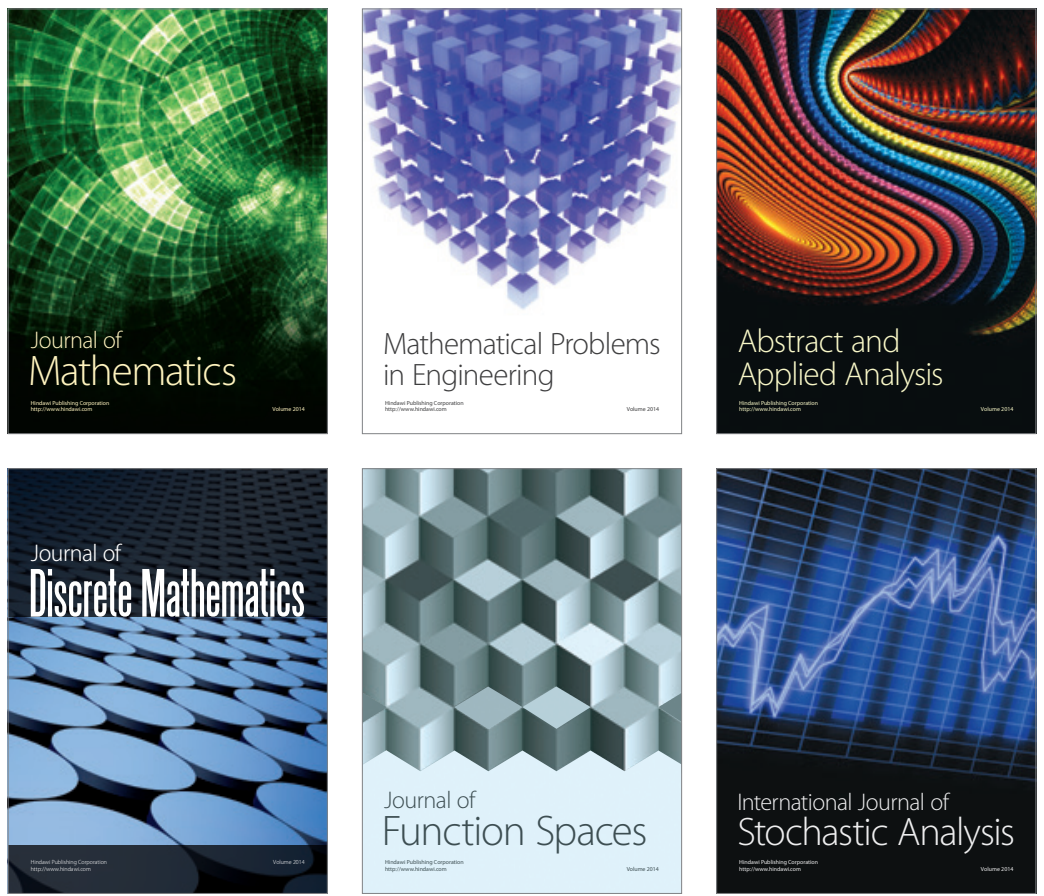

Journal of

Function Spaces




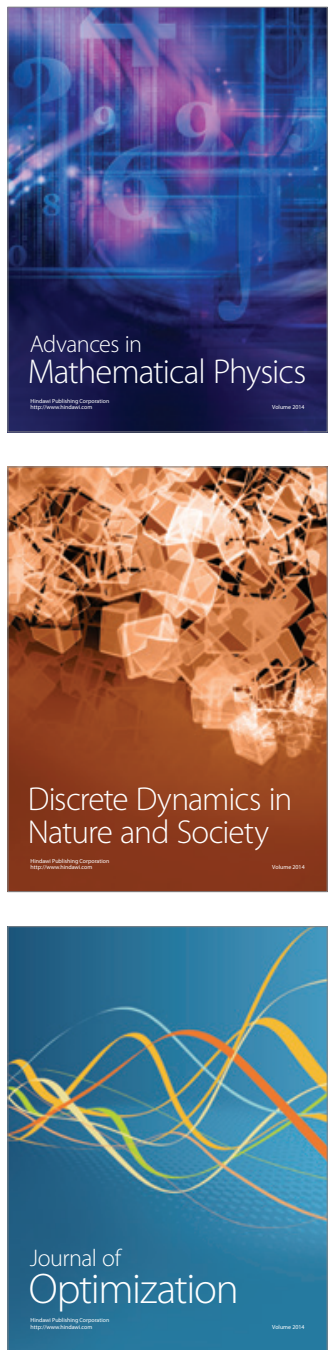